\title{
Percutaneous Transhepatic Cholangioscopy and Lithotripsy for Intrahepatic Biliary Stones and Sclerosing Cholangitis Secondary to Kasai Procedure for Biliary Atresia: A Case Report
}

\author{
Gkekas Ioannis $^{1, ~ *}$, Fagerström Niklas ${ }^{1}$, Waldthaler Alexander ${ }^{2}$, Laustsen Jesper ${ }^{2}$, Arnelo Urban ${ }^{1}$ \\ ${ }^{1}$ Department of Surgery, Karolinska Institute at Huddinge University Hospital, Stockholm, Sweden \\ ${ }^{2}$ Functional Unit GI Endoscopy, Karolinska Institute, Stockholm, Sweden
}

Email address:

ioannis.gkekas@sll.se (G. Ioannis)

${ }^{*}$ Corresponding author

\section{To cite this article:}

Gkekas Ioannis, Fagerström Niklas, Waldthaler Alexander, Laustsen Jesper, Arnelo Urban. Percutaneous Transhepatic Cholangioscopy and Lithotripsy for Intrahepatic Biliary Stones and Sclerosing Cholangitis Secondary to Kasai Procedure for Biliary Atresia: A Case Report. Journal of Surgery. Vol. 7, No. 2, 2019, pp. 41-45. doi: 10.11648/j.js.20190702.14

Received: April 1, 2019; Accepted: April 23, 2019; Published: May 20, 2019

\begin{abstract}
The primary therapy for biliary atresia is a surgical hepatic portoenterostomy, known as Kasai procedure, which has been shown to reduce mortality. Patients that undergo this procedure often develop complications such as ascending cholangitis and biliary cirrhosis. In case of recurrent cholangitis, surgical revision and percutaneous biliary drainage are the most used techniques, while in rare cases endoscopic retrograde cholangiopancreatography (ERCP) has been used. Here we report a case of a patient with intrahepatic lithiasis and secondary sclerosing cholangitis after Kasai procedure, successfully treated with percutaneous transhepatic cholangioscopy (PTCS)-assisted lithotripsy.
\end{abstract}

Keywords: Biliary Atresia, Kasai Procedure, Lithotripsy, Sclerosing Cholangitis, Case Report

\section{Introduction}

Biliary atresia (BA) is a disorder of infants characterized by obliteration or discontinuity of the extrahepatic biliary system, resulting in obstruction of bile flow. Untreated, the resulting cholestasis leads to progressive conjugated hyperbilirubinemia, cirrhosis, and hepatic failure [1]. BA has an incidence of approximately one in 10,000 live births worldwide [2]. The Kasai procedure (hepatic portoenterostomy) is the primary treatment for infants with BA. The surgery is performed by creating an anastomosis between a Roux-en-Y loop of bowel and the liver hilum, to restore bile flow to the small bowel [2]. Early postoperative complications include: cholangitis, bleeding, leak from anastomosis, prolonged ileus, and intestinal obstruction. Late complications include: cessation of bile flow, recurrent cholangitis, portal hypertension, ascites, hepatopulmonary syndrome, formation of bile lakes in the liver, and cirrhosis [2, 3]. The treatment previously used for recurrent cholangitis caused by biliary obstruction has included surgical revision, percutaneous biliary drainage and, in some rare cases, even endoscopic retrograde cholangiopancreatography (ERCP). Recently, however, the introduction of percutaneous transhepatic cholangiopscopy (PTCS) has improved access to the biliary system in those cases in which a peroral endoscopic approach to the biliary system is difficult or impossible [4, 5]. Multiple series using these procedures have demonstrated substantial improvements in mortality, but long-term complications remain a significant problem, with most patients developing cholangitis and liver failure requiring liver transplantation [6]. Here we report the use of PTCS in a patient with recurrent cholangitis, intrahepatic lithiasis and sclerosing cholangitis after a Kasai procedure. For this patient, a previous ERCP attempt had failed to clear the biliary obstruction.

\section{Case Report}

A 25-year-old woman was admitted to our hospital (Huddinge University Hospital, Stockholm, Sweden) for her 
third episode of cholangitis over the last year. She had undergone hepatic portoenterostomy at 12 weeks of age because of BA. After the operation, the patient was generally feeling well, except for episodes of sporadic cholangitis occurring approximately once a year. However, during the last year the patient started developing recurrent cholangitis necessitating repeated courses of intravenous antibiotics and hospitalization. A liver biopsy, taken one year before admission to our hospital, showed no signs of hepatic disease. However, despite medical therapy with ursodesoxycholic acid, a magnetic resonance cholangiopancreatography (MRCP) revealed signs of intrahepatic strictures, as in sclerosing cholangitis, and multiple intrahepatic lithiasis with calculi up to $2 \mathrm{~cm}$. No signs of cirrhosis or portal hypertension were observed. After discussing the case in a multidisciplinary conference, the team decided to treat the intrahepatic stones via double balloon ERCP, due to the previous Roux-en-Y reconstruction. Despite the successful double balloon enteroscopy, which gave access to the portoenterostomy allowing cannulation and complete cholangiogram of the left biliary tract, no cannulation could be achieved for the right biliary tract, regardless of the use of small caliber and swing tip catheters. In fact, most of the calcification and a 2-cm stone were found in the right side of the biliary tract (Figure 1). Access to the right biliary tract was achieved by using a percutaneous transhepatic cholangiography (PTC). Once there, a 10-Fr catheter was placed so that free bile could flow down to the intestinal loop (Figure 2). A secondary percutaneous transhepatic lithotripsy was performed to the 2-cm stone in the right bile system and the stone was removed with the extraction balloon. In addition, supplementary dilation of the right biliary system was performed, and a 14-Fr biliary drainage was inserted. The patient recovered well after the PTCS/lithotripsy, without any sign of cholangitis. A third PTCS, performed 4 weeks later, confirmed the complete clearance of the right biliary tract (Figures 3, 4). In addition, further biopsies taken during this PTCS confirmed the absence of malignancy. Subsegmental cholangioscopic access was not achieved during any PTCS session. Therefore, the patient is planned to undergo further bile tract visualization with a new MRCP, in order to confirm full subsegmental stone clearance.

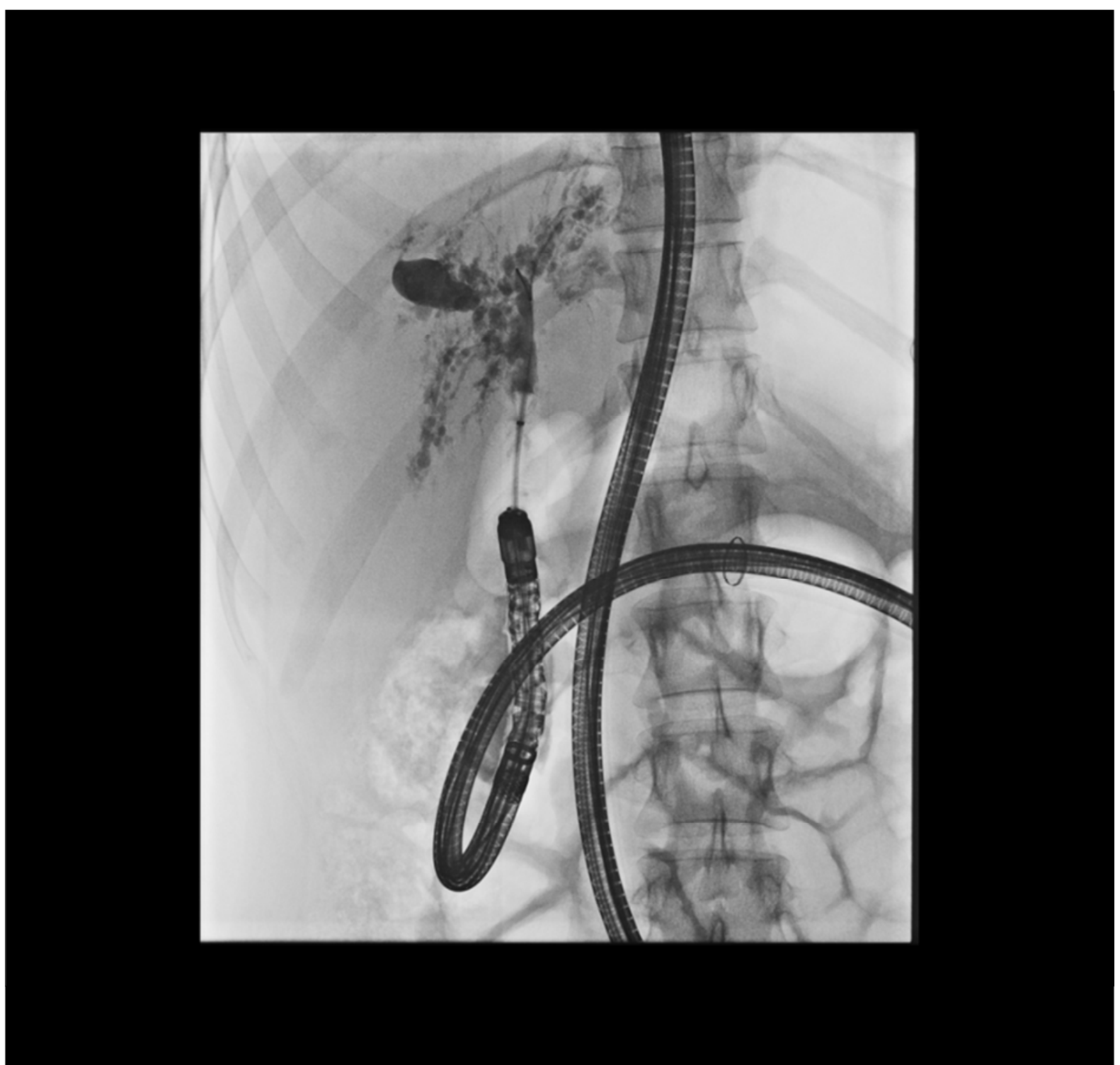

Figure 1. ERCP cannulation in the left hepatic duct, obstruction due to lithiasis in the right duct. 


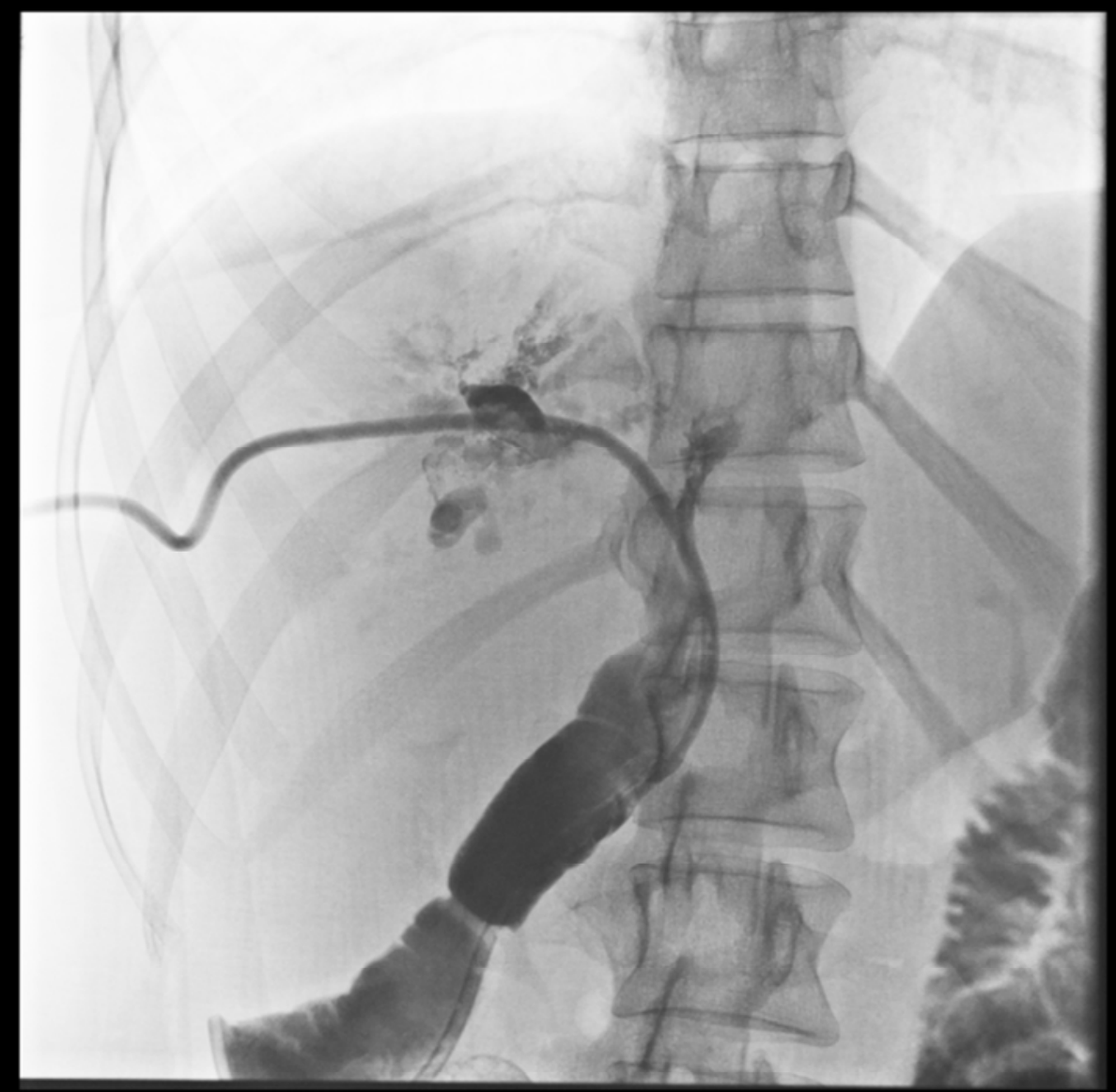

Figure 2. Access to the right hepatic duct through PTC.

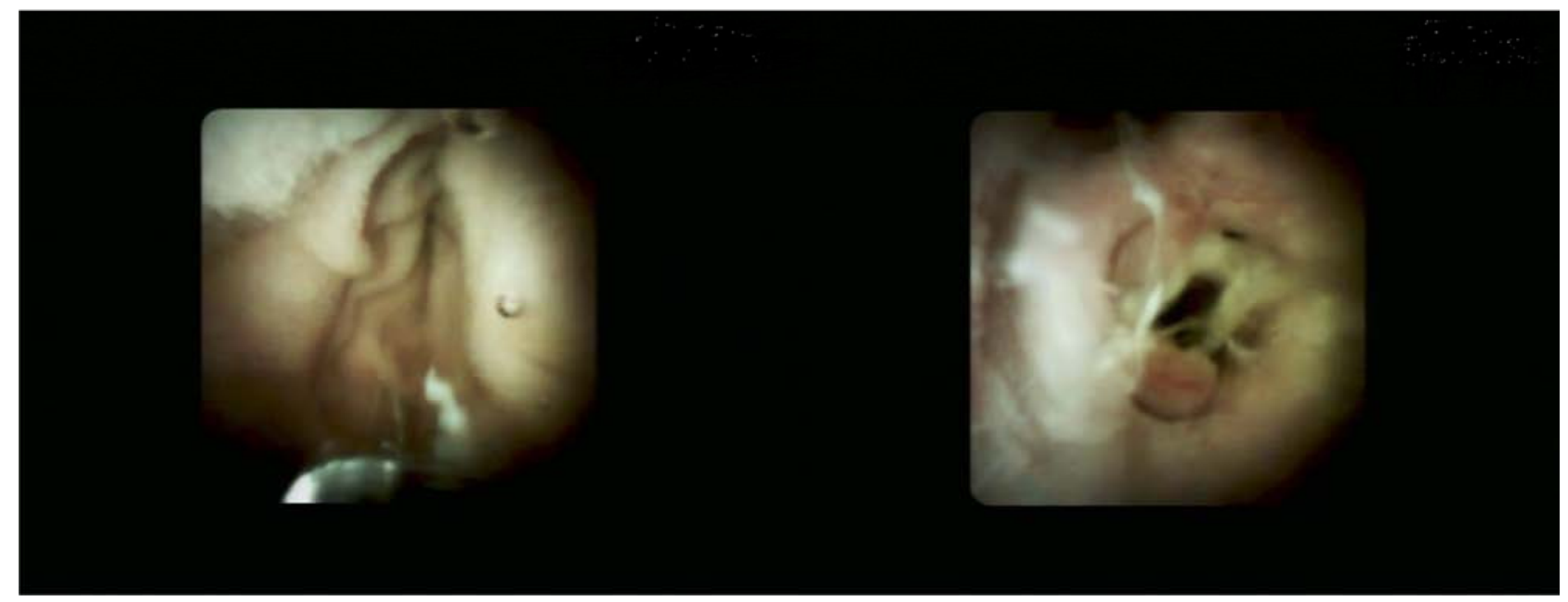

Figure 3. Percutaneous cholangioscopy with complete clearance of the right hepatic duct. 


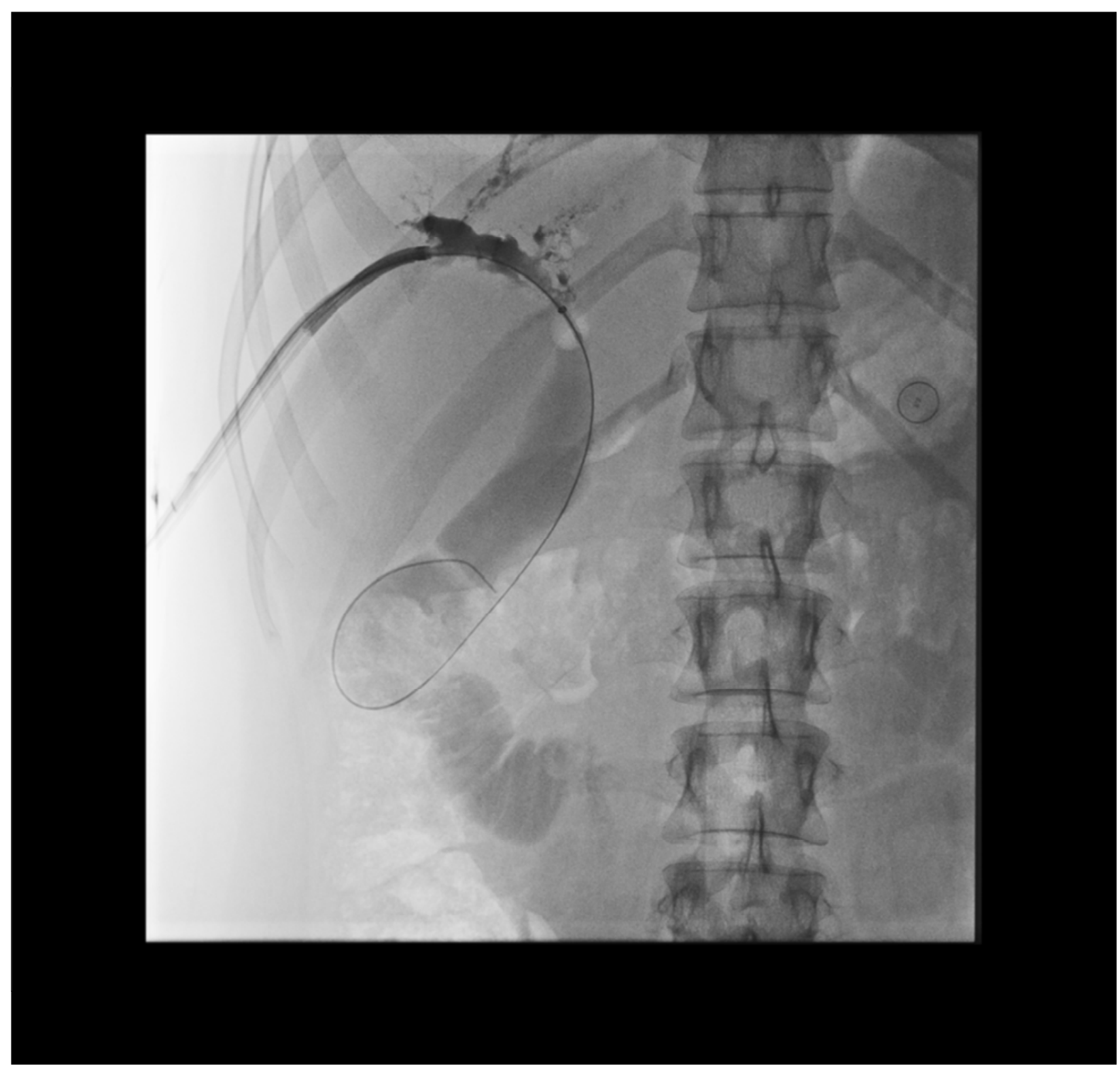

Figure 4. Balloon cholangiography, complete clearance.

\section{Discussion}

Secondary cholangitis is a common complication in patients with hepatic portoenterostomy. Two of the mechanism that have been proposed to cause this complication are intestinal bacterial stasis and incomplete biliary drainage. Patients with secondary cholangitis often have poor outcomes, progression of portal hypertension and the need of early transplantation [7, 8]. Both the use of antibiotics for intestinal decontamination and that of PTC or surgical revision for mechanical relief of biliary obstruction have been previously reported for this condition [6]. Additionally, cases of endoscopic treatment with the use of balloon-assisted ERCP have also been reported $[5,6]$. However, in those cases in which patients are unsuitable for surgery, or those in which balloon-assisted ERCP fails to establish bile cannulation, PTCS may be the optimal alternative both as diagnostic tool and as therapeutic solution, as described in a few rare cases of PTCS-assisted lithotripsy [9]. To our knowledge, this is the first report of percutaneous transhepatic lithotripsy in a patient with sclerosing cholangitis and biliary stones secondary to Kasai procedure. This technique is effective, safe, and has a low complication rate. Also, the combined use of endoscopic procedures (Rendezvous) increases the possibility of complete bile clearance [10]. Percutaneous transhepatic treatment of anastomotic strictures is a safe and highly effective technique for achieving internal biliary drainage, especially for patients with anastomotic dysfunctions after hepaticojejunostomy. Also, temporary transhepatic intubation seems to be a promising minimally invasive alternative to surgery [11]. Current studies have been demonstrate the advantages of PTCS-assisted lithotripsy on cases of calculus in the right hepatic lobe, bilateral lobes, or in patients with hepatic function at Child-Pugh stage $\mathrm{C}$ and intolerant to conventional 
surgery. However, the use of these techniques, particularly in patients with classic BA, are recommended in highly specialized centers. Meticulous patient selection and a cooperative team approach are generally suggested in order to minimize the incidence of complications and to provide effective management [11].

\section{Conclusion}

Percutaneous transhepatic lithotripsy in patients with sclerosing cholangitis and biliary stones secondary to Kasai procedure is challenging but safe and effective technique in highly specialized centers. For patients with anastomotic dysfunctions after hepaticojejunostomy who are unsuitable for surgery, or those in which balloon-assisted ERCP fails to establish bile cannulation, PTCS-assisted lithotripsy can be the optimal alternative. Patients selection and an experienced cooperative team can minimize the complication rate both as diagnostic tool and as therapeutic solution.

\section{References}

[1] Wildhaber BE. Biliary atresia: 50 years after the first Kasai. ISRN surgery 2012; 2012:132089 doi: 10.5402/2012/132089.

[2] Sinha CK and Davenport M. Biliary atresia. J Indian Assoc Pediatr Surg 2008; 13 (2):49-56.

[3] Kim, Seong Hyun, et al. "Percutaneous cholangioscopic lithotripsy for afferent loop syndrome caused by enterolith development after Roux-en-Y hepaticojejunostomy: a case report." Clinical endoscopy 46.6 (2013): 679.
[4] Orman ES, Miller CB, Grimm IS, and Barritt AS Single Balloon Enteroscopy-Assisted ERCP for Treatment of Cholangitis in a Patient with a Kasai Portoenterostomy. $J$ Pediatr Surg 2012; 47 (5): E1-5.

[5] Zhang, Zongming, et al. "Strategies of minimally invasive treatment for intrahepatic and extrahepatic bile duct stones." Frontiers of medicine 11.4 (2017): 576-589.

[6] Du L, D'Souza P, Thiesen A, et al. Percutaneous transhepatic cholangioscopy for indeterminate biliary strictures using the SpyGlass system: a case series. Endoscopy 2015; 47 (11): 1054-6.

[7] Sundaram SS, Mack CL, Feldman AG and Sokol RJ. Biliary atresia: Indications and timing of liver transplantation and optimization of pretransplant care. Liver Transpl 2017; 23 (1): 96-109.

[8] Wang P, Sun B, Huang B, et al. Comparison between percutaneous transhepatic rigid cholangioscopic lithotripsy and conventional percutaneous transhepatic cholangioscopic surgery for hepatolithiasis treatment. Surg laparosc endosc percutan tech 2016; 26 (1): 54-9.

[9] Hamada T, Tsujino T, Sasahira N, et al. Percutaneous transhepatic cholangioscopy with an ultraslim video upper endoscope with $\mathrm{CO} 2$ insufflation: a feasibility study. Gastroint endosc 2011; 74 (3): 696-699.

[10] Schumacher B, Othman T, Jansen M, et al. Long-term followup of percutaneous transhepatic therapy (PTT) in patients with definite benign anastomotic strictures after hepaticojejunostomy. Endoscopy 2001; 33 (5): 409-415.

[11] Oh HC, Lee SK, Lee TY, et al. Analysis of percutaneous transhepatic cholangioscopy-related complications and the risk factors for those complications. Endoscopy 2007; 39 (8): 731-736. 